\title{
Microplasma Noise Stimulated by Microwave Electric Field
}

\author{
A. Namajūnas ${ }^{a}$, A. Tamaševičius ${ }^{a, *}$, G. Mykolaitis ${ }^{a, b}$, \\ S. Bumelient $\dot{E}^{a}$ AND J. PoŽElA ${ }^{a}$ \\ ${ }^{a}$ Semiconductor Physics Institute, A. Goštauto 11, Vilnius, 01108, Lithuania \\ ${ }^{b}$ Department of Physics, Faculty of Fundamental Sciences
}

Vilnius Gediminas Technical University, Saulètekio 11, Vilnius 10223, Lithuania

\begin{abstract}
$\mathrm{Si}$ and GaAs avalanche diodes containing microplasmas are investigated. Microwave field applied to the diode in addition to reverse dc bias results in considerable spread of noise spectrum and in the increase of noise power. The microplasma noise spectra cover very high (30 to $300 \mathrm{MHz}$ ) and ultrahigh (300 to $1000 \mathrm{MHz}$ ) frequency bands, while the effective noise temperature is about $10^{8} \mathrm{~K}$.
\end{abstract}

PACS numbers: 05.40.Ca, 52.25.Gj, 72.70.+m, 85.30.- $\mathrm{Z}$

\section{Introduction}

In semiconductors the impurities tend to concentrate around dislocations. When a high electric field is applied, tiny domains of enhanced impact ionization, called microplasmas, appear in these areas [1]. Due to small dimensions $\left(\approx 1 \mu \mathrm{m}^{3}\right)$ of microplasmas fluctuations of the number of the carriers result in chaotic bursts and extinctions of the avalanche. The current through the microplasma looks like a sequence of rectangular pulses of fixed height, typically 50 to $100 \mu \mathrm{A}$. Meanwhile the lengths of the pulses and pauses between them vary in a random manner. The pulsed current exhibits typical features of noise, namely non-periodic time series and broadband continuous power spectra. Microplasma noise is commonly observed in the reverse-biased $p-n$ junctions at the onset of the avalanche breakdown. This type of noise was first described in details 50 years ago [2]. For the 35 successive years the phenomenon was studied worldwide, both experimentally and theoretically. Many of the characteristics of microplasma noise have been considered and a statistical model has been generalized in [3].

*corresponding author; e-mail: tamasev@pfi.lt 
To our best knowledge microplasma noise was investigated experimentally under the dc conditions. An exception was a short communication [4] where apart from dc bias an additional RF (50 MHz) voltage was applied to a silicon avalanche diode [5]. This RF perturbation yielded a significant spread of noise spectrum, specifically from several megahertz to $50 \mathrm{MHz}$, while the spectral density remained almost the same. Consequently the noise power has increased considerably. In the present paper we describe the effect of enhancement of the microplasma noise in the microwave range $(>300 \mathrm{MHz})$.

\section{Experimental results}

The experimental setup is sketched in Fig. 1 (left). The dc bias voltage $V_{0}$ and the amplitude $V_{1 A}$ of the RF perturbation satisfied the following conditions: $V_{1 A} \ll V_{0}<V_{\text {th }},\left(V_{1 A}+V_{0}\right)>V_{\text {th }}$ (here $V_{\text {th }}$ is the threshold avalanche breakdown voltage of the diode). Experiments have been carried out at room temperature
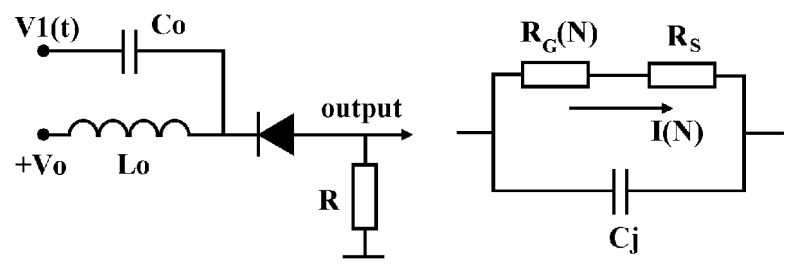

Fig. 1. Experimental setup (left) and equivalent circuit of the diode with microplasma (right). The Co and the Lo are the blocking elements. $V_{1}(t) \equiv V_{1 A} \sin \left(2 \pi f_{1} t\right)$.

using $\mathrm{Si}$ and GaAs avalanche diodes with $V_{\mathrm{th}} \approx 18 \mathrm{~V}$. The diode capacitances are $C_{j} \approx 3 \mathrm{pF}$, and $C_{j} \approx 1 \mathrm{pF}$, respectively, and the load resistance $R=50 \Omega$. Noise spectra are presented in Fig. 2.
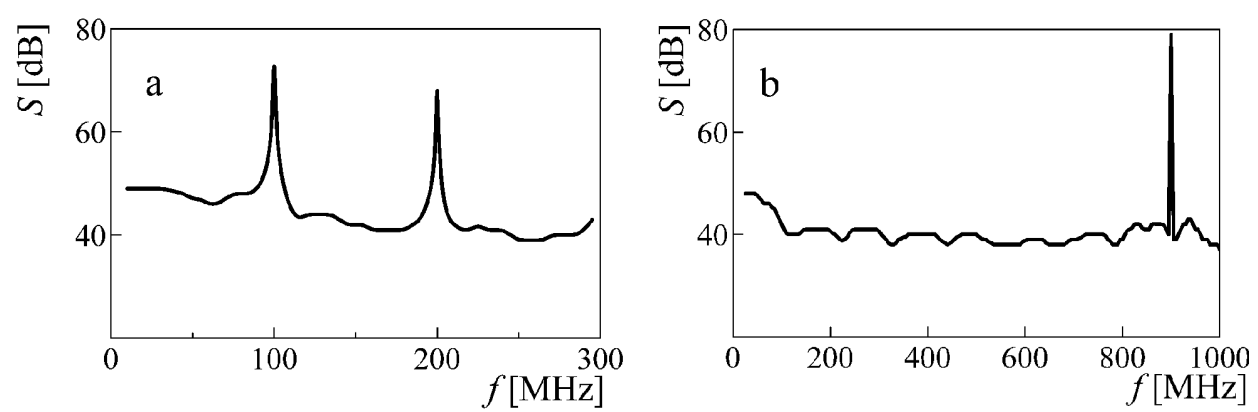

Fig. 2. Experimental noise spectra. (a) Si diode, $V_{0}=17.5 \mathrm{~V}, V_{1 A}=0.2 \mathrm{~V}, f_{1}=$ $100 \mathrm{MHz}$. (b) GaAs diode, $V_{0}=17.5 \mathrm{~V}, V_{1 A}=0.5 \mathrm{~V}, f_{1}=900 \mathrm{MHz}$. Spectral resolution $\Delta f=120 \mathrm{kHz}$. The level of $0 \mathrm{~dB}$ corresponds to an effective noise temperature of $10^{4} \mathrm{~K}$. 


\section{Mathematical model and numerical results}

Let us adapt the statistical model presented in [3] to the equivalent circuit of the diode shown in Fig. 1 (right). For low load resistance $R \ll R_{G}(N)$, $R_{S},\left(2 \pi f_{1} C_{j}\right)^{-1}$ the voltage across the diode is just the external applied voltage $V=V_{0}+V_{1 A} \sin \left(2 \pi f_{1} t\right)$. The resistance of the avalanche area can be approximated as $R_{G}(N)=R_{1} / N$, where $N$ is the number of carriers in the microplasma and $R_{1} \equiv R_{G}(1)$. Then the voltage drop across $R_{G}$ and current through microplasma are given by

$$
V_{G}(t)=\frac{V}{1+\varepsilon N(t)}, \quad I(t)=\frac{V}{R_{1}} \cdot \frac{N(t)}{1+\varepsilon N(t)} \cong \frac{V}{R_{1}} N(t), \quad \varepsilon=\frac{R_{S}}{R_{1}} .
$$

Here $R_{S}$ represents all the effects that damp the avalanche multiplication of the carriers, e.g. the spreading resistance, self-heating of microplasma, etc. Instead of continuous time function $N(t)$ it is convenient to consider the evolution of "generations", that is the discrete time sequence $N_{k}=N(k \tau)$, where $\tau$ is the transit time through the microplasma, and $k=0,1,2, \ldots$ is a serial of the generation. The probability of emergence of $m_{k}$ primary carriers during time interval $\tau$ also the probability of producing $n_{i}$ secondary carriers by the $i$-th primary one are described by the following Poisson distributions:

$$
\begin{aligned}
& P\left(m_{k}, \tau\right)=\frac{\exp (-\nu \tau)(\nu \tau)^{m_{k}}}{m_{k} !}, \quad m_{k}=0,1,2 \ldots \\
& P\left(n_{i}\right)=\frac{\exp (-\gamma) \gamma^{n_{i}}}{n_{i} !}, \quad n_{i}=0,1,2 \ldots
\end{aligned}
$$

In (3) $\nu$ is the average rate of emergence of carriers in the microplasma area due to thermal generation, tunneling, etc., $\gamma$ is the average number of ionization impacts (for simplicity $\gamma$ is assumed to be the same for electrons and holes). The total number of carriers in the $(k+1)$ th generation is given by

$$
N_{k+1}=\sum_{i=1}^{m_{k}+N_{k}} n_{i} \quad\left(k=0,1,2 \ldots, \quad N_{0}=0\right) .
$$

The parameter $\gamma$ exponentially depends on the overvoltage $V_{G}-V_{\mathrm{th}}$ :

$$
\gamma=\exp \left(b \frac{V_{G}-V_{\mathrm{th}}}{V_{\mathrm{th}}}\right) .
$$

The coefficient $b$ depends on physical parameters of the semiconductor. At $V_{G}>V_{\mathrm{th}}$ the parameter $\gamma>1$. Therefore the number of carriers in the subsequent generations $N_{k}$ would grow unlimited. Repartition of the applied voltage $V$ between the resistors $R_{G}$ and $R_{S}$ limits the growth: $V_{G} \rightarrow V_{\mathrm{th}}$ and $\gamma \rightarrow 1$. The number of carriers $N_{k}$ oscillates randomly around some value $N_{S}$ corresponding to $V_{G}\left(N_{S}\right)=V_{\text {th. }}$. Meanwhile, some fluctuations can lead to $N_{k}=0$, that is to the off state. 

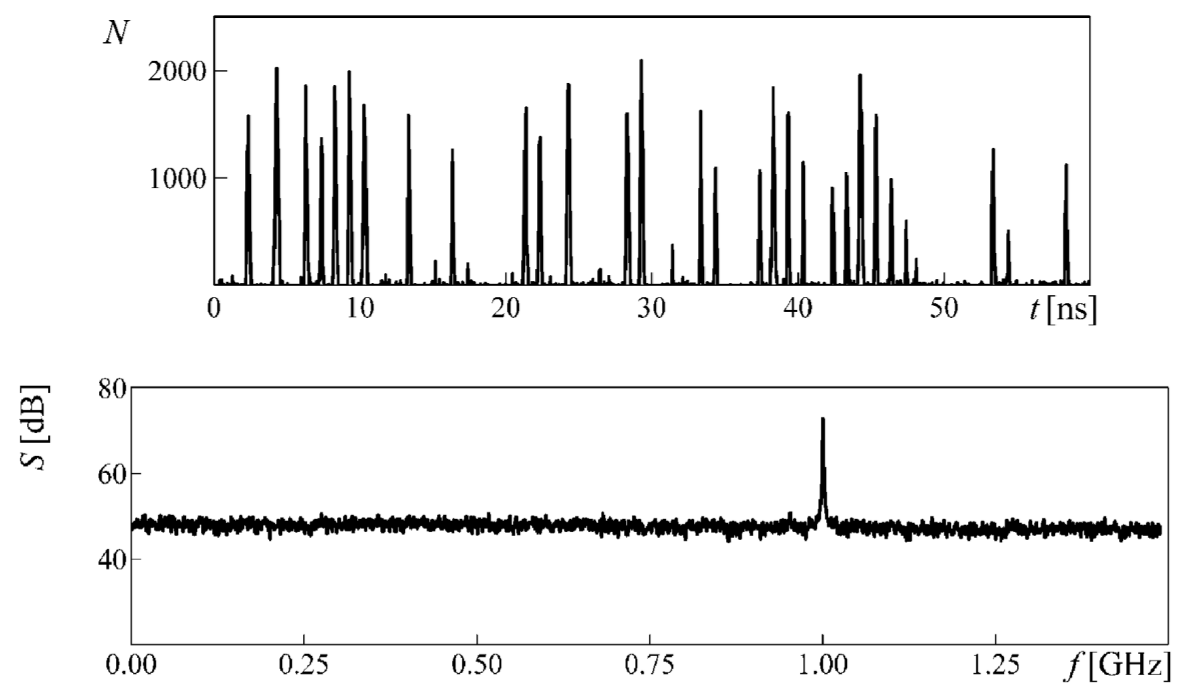

Fig. 3. Calculated evolution of $N(t)$ (top), power spectrum of the output signal (bottom). $V_{0}=17.8 \mathrm{~V}, V_{1 A}=0.5 \mathrm{~V}, f_{1}=10^{9} \mathrm{~Hz}$.

Employing the relations (1)-(4) and using the following parameters: $V_{\mathrm{th}}=$ $18 \mathrm{~V}, R_{S}=10^{3} \Omega, R_{1}=10^{8} \Omega, \tau=3 \times 10^{-12} \mathrm{~s}, \nu=10^{10} \mathrm{~s}^{-1}, b=10$ the evolution of $N_{k}$ has been simulated and the power spectrum of the output signal $V_{R}(t)=I(t) R \propto N(t)$ has been calculated (Fig. 3).

\section{Conclusions}

Avalanche diodes with microplasmas biased close to the breakdown voltage and stimulated by microwave electric field demonstrate a considerable spread of microplasma noise spectrum, thus covering very high frequency (30 to $300 \mathrm{MHz}$ ) and ultrahigh frequency (300 to $1000 \mathrm{MHz}$ ) bands. The effective noise temperature goes up to $10^{8} \mathrm{~K}$.

\section{References}

[1] J. Pozhela, Plasma and Current Instabilities in Semiconductors, Pergamon Press, Oxford 1981.

[2] K.G. McKay, Phys. Rev. 94, 877 (1954).

[3] A.M. Namajūnas, J.K. Požela, A.V. Tamaševičius, Fiz. Tekhn. Poluprovod. 23, 1606 (1989).

[4] A.M. Namajūnas, J.K. Požela, A.V. Tamaševičius, Doklady AN SSSR 307, 102 (1989).

[5] V.K. Aladinsky, V.I. Dashin, A.S. Sushchik, A.M. Timerbulatov, Radiotekh. Elektron. 18, 342 (1973). 